\title{
FAST COMIPUTATION OF PERIODIC CONTINUED FRACTIONS
}

\author{
Kuo-Liang CHUNG, Wen-Chin CHEN and Ferng-Ching LIN \\ Department of Computer Science and Information Engineering, Nctional Taiwan University, Taipei, Taiwan 10764, Taiwan \\ Communicated by David Gries \\ Received 1 June 1989 \\ Revised 3 July 1989
}

Periodic continued fractions are useful for represerting or approximating numbers. Based on a self-substituticn cczcept, we derive an $\mathrm{O}(\log n)$ algorithm to compute periodic continued fractions. Two application exampies, the apFroxitiation of a quadratic surd inumbéi alıd ilie soiving of second-order linear recurrence, are presented.

Keywords: Design of algorithms, periodic continued fraction, self-substitution, second-order linear recurrence, Fibonacci number

\section{i. Introduction}

The following form represents a continued fraction $(\mathrm{CF})$ :

$$
V_{n}=a_{1}+\frac{b_{2}}{a_{2}+\frac{b_{3}}{\ldots \overline{a_{n-1}+\frac{b_{n}}{a_{n}}}}}
$$

The CFs provide a useful means for solving equations such as three-term recurrence and Recatti equation and for approximating numbers such as $\mathrm{e}, \pi, \arctan (x)[2,6,7]$.

The above representation for $\mathrm{CF}$ is typographically cumbersome and can be replaced by the more compact form:

$$
V_{n}=a_{1}+\frac{b_{2}}{a_{2}+} \frac{b_{3}}{a_{3}+\cdots} \frac{b_{n}}{a_{n}}
$$

A CF with period $p$ can be expressed as

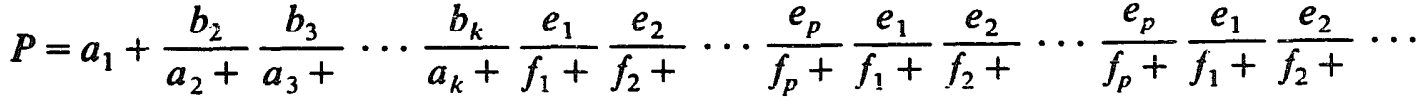

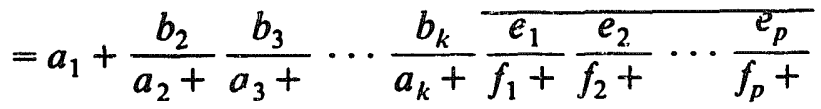

for some constant $k$. 
In this paper, we first introduce a substitution scheme as the base for designing fast computations of CFs. Then we embed a self-substitution concept into the scheme to design an $O(\log n)$ algorithm for computing any periodic CF. Two examples, the approximation of $\sqrt{7}$ and the solving of second-order linear recurrence, are used to illustrate the application of our method.

\section{The substitution scheme}

Consider the iroblem of computing $V_{n}$ in equation (1). At first glance, it seems that the rational form of $V_{\text {s }}$ caninot be efficiently obtained because there exists a dependence relation in the stair-like, bottom-up computations of $V_{n}$. Fortunately, the dependence relation can be resolved by decomposing $V_{n}$ into disjoint sub-CFs that can be processed separately. For simplicity, we use $V_{4}$ to illustrate this idea. $V_{4}$ can be decomposed into four sub-CFs:

$$
V_{4}=a_{1}+X_{3}, \quad X_{3}=\frac{b_{2}}{a_{2}+X_{2}}, \quad X_{2}=\frac{b_{3}}{a_{3}+X_{1}}, \quad X_{1}=\frac{b_{4}}{a_{4}}
$$

Each of the right-hand sides of the equations in (3) is a sub-CF. The pseudo-variables $X_{3}, X_{2}$ and $X_{1}$ serve as the substitution bridges without any functional meaning.

Let the general rational form $\left(c_{1}+c_{2} X_{i}\right) /\left(c_{3}+c_{4} X_{i}\right)$ be represented by the notation $\left(c_{1}, c_{2}, c_{3}, c_{4}, X_{i}\right)$. The equations in (3) can be iewritten as:

$$
\begin{array}{ll}
V_{4}=\frac{a_{1}+X_{3}}{1}=\left(a_{1}, 1,1,0, X_{3}\right), & X_{3}=\frac{b_{2}}{a_{2}+X_{2}}=\left(b_{2}, 0, a_{2}, 1, X_{2}\right), \\
X_{2}=\frac{b_{3}}{a_{3}+X_{1}}=\left(b_{3}, 0, a_{3}, 1, X_{1}\right), & X_{1}=\frac{b_{4}}{a_{4}+X_{0}}=\left(b_{4}, 0, a_{4}, 1, X_{0}\right),
\end{array}
$$

where the pseudo-variable $X_{0}$ will be replaced by zero after all the substitution operations are completed.

Since substituting $X_{i}=\left(d_{1}+d_{2} X_{j}\right) /\left(d_{3}+d_{4} X_{j}\right)$ into $\left(c_{1}+c_{2} X_{i}\right) /\left(c_{3}+c_{4} X_{i}\right)$ gives

$$
\left(\left(c_{1} d_{3}+c_{2} d_{1}\right)+\left(c_{1} d_{4}+c_{2} d_{2}\right) Y_{j}\right) /\left(\left(c_{3} d_{3}+c_{4} d_{1}\right)+\left(c_{3} d_{4}+c_{4} d_{2}\right) X_{j}\right),
$$

a substitution operation - with eight multiplications and four additions can be defined as

$$
\begin{aligned}
& \left(c_{1}, c_{2}, c_{3}, c_{4}, X_{i}\right) \circ\left(d_{1}, d_{2}, d_{3}, d_{4}, X_{j}\right) \\
& \quad=\left(c_{1} d_{3}+c_{2} a_{1}^{\prime}, c_{1} d_{4}+c_{2} a_{2}^{\prime}, c_{3} d_{3}+c_{4} d_{1}, c_{3} d_{4}+c_{4} d_{2}, X_{j}\right) .
\end{aligned}
$$

\section{Lemma 2.1. Substitution operation • is associative.}

Proof. For any $\left(c_{1}, c_{2}, c_{3}, c_{4}, X_{i}\right),\left(d_{1}, d_{2}, d_{3}, d_{4}, X_{j}\right)$ and $\left(e_{1}, e_{2}, e_{3}, e_{4}, X_{k}\right)$, we have

$$
\begin{aligned}
{\left[\left(c_{1}, c_{2}, c_{3}, c_{4}, X_{i}\right) \circ\left(d_{1}, d_{2}, d_{3}, c_{4}^{\prime}, X_{j}\right)\right] \circ\left(e_{1}, e_{2}, e_{3}, e_{4}, X_{k}\right) } \\
=\left(c_{1} d_{3}+c_{2} d_{1}, c_{1} d_{4}+c_{2} d_{2}, c_{3} d_{3}+c_{4} d_{1}, c_{3} d_{4}+c_{4} d_{2}, X_{j}\right) \circ\left(e_{1}, e_{2}, e_{3}, e_{4}, X_{k}\right) \\
=\left(c_{1} d_{3} e_{3}+c_{2} d_{1} e_{3}+c_{1} d_{4} e_{i}+c_{2} d_{2} e_{1}, c_{1} d_{3} e_{4}+c_{2} d_{1} e_{4}+c_{1} d_{4} e_{2}+c_{2} d_{2} e_{2},\right. \\
\\
\left.\quad c_{3} d_{3} e_{3}+c_{4} d_{1} e_{3}+c_{3} d_{4} e_{1}+c_{4} d_{2} e_{1}, c_{3} d_{3} e_{4}+c_{4} d_{1} e_{4}+c_{3} d_{4} e_{2}+c_{4} d_{2} e_{2}, X_{k}\right)
\end{aligned}
$$




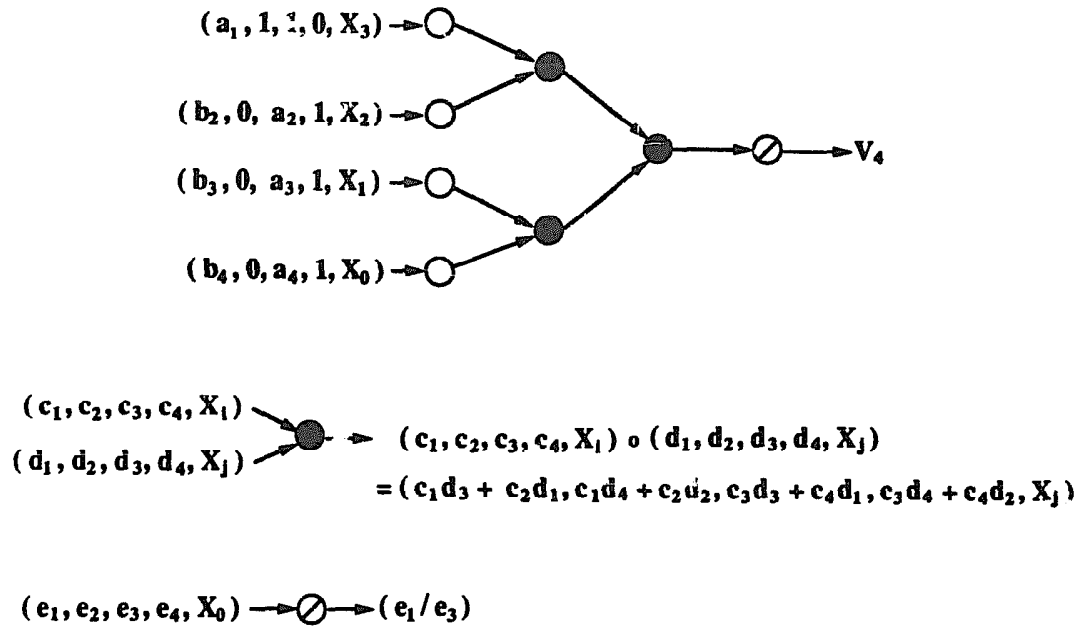

Fig. 1. A computation tree of $V_{4}$.

and

$$
\begin{aligned}
\left(c_{1}, c_{2}, c_{3}, c_{4}, X_{i}\right) \circ\left[\left(d_{1}, d_{2}, d_{3}, d_{4}, X_{j}\right) \circ\left(e_{1}, e_{2}, e_{3}, e_{4}, X_{k}\right)\right] \\
=\left(c_{1}, c_{2}, c_{3}, c_{4}, X_{i}\right) \circ\left(d_{1} e_{3}+d_{2} e_{1}, d_{1} e_{4}+d_{2} e_{2}, d_{3} e_{3}+d_{4} e_{1}, d_{3} e_{4}+d_{4} e_{2}, X_{k}\right) \\
=\left(c_{1} d_{3} e_{3}+c_{2} d_{1} e_{3}+c_{1} d_{4} e_{1}+c_{2} d_{2} e_{1}, c_{1} d_{3} e_{4}+c_{2} d_{1} e_{4}+c_{1} d_{4} e_{2}+c_{2} d_{2} e_{2},\right. \\
\\
\left.\quad c_{3} d_{3} e_{3}+c_{4} d_{1} e_{3}+c_{3} d_{4} e_{1}+c_{4} d_{2} e_{1}, c_{3} d_{3} e_{4}+c_{4} d_{1} e_{4}+c_{3} d_{4} e_{2}+c_{4} d_{2} e_{2}, X_{k}\right) .
\end{aligned}
$$

Both computation sequences give the same rational form.

By Lemma 2.1, $V_{4}=\left(a_{1}, 1,1,0, X_{3}\right) \circ\left(b_{2}, 0, a_{2}, 1, X_{2}\right) \circ\left(b_{3}, 0, a_{3}, 1, X_{1}\right) \circ\left(b_{4}, 0, a_{4}, 1, X_{0}\right)$ can be computed in any order. Fig. 1 shows a computation tree for $V_{4}$. In Fig. 1 , the white nodes at ievel 1 transmit data without changing them. The black nodes at levels 2 and 3 perform the function defined by substitution operation $\circ$. The slashed node at level 4 assigns zero to the pseudo-variable $X_{0}$ to obtain the desired result.

\section{The algorithm}

The periodic CF in (2) can be partitioned into the following sub-CFs:

$$
\begin{aligned}
& P_{n}=a_{1}+\frac{b_{2}}{a_{2}+} \frac{b_{3}}{a_{3}+} \cdots \frac{b_{k}}{a_{k}+X_{((n-k) / p)}}, \\
& X_{[(n-k) / p]}=\frac{e_{1}}{f_{1}}+\frac{e_{2}}{f_{2}+} \cdots \frac{e_{p}}{f_{p}+X_{(n-k) ; p]-1}}, \\
& X_{\mid(n-k) / p !-1}=\frac{e_{1}}{f_{1}+} \frac{e_{2}}{f_{2}+} \cdots \frac{e_{p}}{f_{p}+X_{|(n-k) / p|-2}}, \\
& X_{1}=\frac{e_{1}}{f_{1}+} \frac{e_{2}}{f_{2}+} \cdots \frac{e_{p}}{f_{p}+X_{0}^{\prime}},
\end{aligned}
$$


where

$$
X_{0}^{\prime}= \begin{cases}X_{0} & \text { if }(n-k) / p \text { is an integer; } \\ \frac{e_{1}}{f_{1}+} \frac{e_{2}}{f_{2}+} \cdots \frac{e_{j}}{f_{j}+X_{0}} & \text { otherwise }\end{cases}
$$

and $j=(n-k)-p\lfloor(n-k) / p\rfloor$.

We first deal with the case when $(n-k) / p$ is an integer and a power of 2 . It can be observed that all the sub-CFs in (4), except the first one, have exactly the same form. It is natural and economical to transform just one, say the last sub-CF, to its rational form and let the other inherit the same rational form associated with their own pseudo-variables implicitly. This arrangement of computations is legal since the substitution operation is associative by Lemma 2.1. Thus, (4) becomes

$$
\begin{aligned}
& P_{n}=a_{1}+\frac{b_{2}}{a_{2}+} \frac{b_{3}}{a_{3}+} \cdots \frac{b_{k}}{a_{k}+X_{(n-k) / p}}, \\
& X_{(n-k) / p}=\left(c_{1}, c_{2}, c_{3}, c_{4}, X_{(n-k) / p-1}\right), \\
& X_{(n-k) / p-1}=\left(c_{1}, c_{2}, c_{3}, c_{4}, X_{(n-k) / p-2}\right), \\
& \vdots \\
& X_{1}=\left(c_{1}, c_{2}, c_{3}, c_{4}, X_{0}\right),
\end{aligned}
$$

for some numbers $c_{1}, c_{2}, c_{3}$, and $c_{4}$. It takes only $\mathrm{O}(p)$ time to complete this first stage of computation.

By a similar argument, it is then sufficient to perform the substitution operation on just one pair of the above rational forms, say $\left(c_{1}, c_{2}, c_{3}, c_{4}, X_{1}\right)$ and $\left(c_{1}, c_{2}, c_{3}, c_{4}, X_{0}\right)$. The other pairs inherit the same rational form, so their substitution operation becomes unnecessary and can be eliminated. After that, (5) becomes

$$
\begin{aligned}
& P_{n}=a_{1}+\frac{b_{2}}{a_{2}+} \frac{b_{3}}{a_{3}+} \cdots \frac{b_{k}}{a_{k}+X_{(n-k) / p}}, \\
& X_{(n-k) / p}=\left(d_{1}, d_{2}, d_{3}, d_{4}, X_{(n-k) / p-2}\right), \\
& X_{(n-k) / p-2}=\left(d_{1}, d_{2}, d_{3}, d_{4}, X_{(n-k) / p-4}\right), \\
& \vdots \\
& X_{2}=\left(d_{1}, d_{2}, d_{3}, d_{4}, X_{0}\right),
\end{aligned}
$$

for some numbers $d_{1}, d_{2}, d_{3}$, and $d_{4}$. This special type of substitution, the self-substitution, takes $O(1)$ time. By applying self-s.dbstitution $\log ((n-k) / p)$ times (the height of a computation tree), (5) is reduced to

$$
\begin{aligned}
& P_{n}=a_{1}+\frac{b_{2}}{a_{2}+} \frac{b_{3}}{a_{3}+} \cdots \frac{b_{k}}{a_{k}+X_{(n-k) / p}} \\
& X_{(n-k) / p}=\left(g_{1}, g_{2}, g_{3}, g_{4}, X_{0}\right)=\frac{g_{1}}{g_{3}},
\end{aligned}
$$

for some numbers $g_{1}, g_{2}, g_{3}$, and $g_{4}$.

It takes $O(k)$ time in the final stage to obtain the rational form of $P_{n}$. Totally, $O(p)+O(\log (n-k) / p)$ $+\mathrm{O}(k)=\mathrm{O}(\log n)$ time is required to complete the computation. It is also clear that only $\mathrm{O}(1)$ space is needed in the whols process. 
Next we discuss the second case when $(n-k) / p$ is an integer but not a power of 2 . Let the binary representation of $(n-k) / p$ be $b(q) \ldots b(1) b(0)$, where $b(q)=1$ is the most significant bit. That is, $(n-k) / p=\sum_{i=0}^{q} b(i) 2^{i}$. For convenience, we use a $\Pi$-notation to represent substitution sequences. $X_{(n-k) / p}$ in (5) can be expressed as

$$
X_{(n-k) / p}=\left(\prod_{\substack{i=q \\ b(i)=1}}^{0}\left(c_{1}, c_{2}, c_{3}, c_{4}\right)^{2^{i}}, X_{0}\right) .
$$

For example, if $(n-k) / p=11$ then $b(3) b(2) b(1) b(0)=1011$. Therefore, we have

$$
X_{11}=\left(\left(c_{1}, c_{2}, c_{3}, c_{4}\right)^{8} \circ\left(c_{1}, c_{2}, c_{3}, c_{4}\right)^{2} \circ\left(c_{1}, c_{2}, c_{3}, c_{4}\right)^{1}, X_{0}\right) \text {. }
$$

According to the self-substitution concept and the information $b(q) \ldots b(1) b(0)$, the rational form of $X_{(n-k) / p}$ can be obtained in $\mathrm{O}([\log (n \dot{-} k) / p])$ time using $\mathrm{O}(1)$ space.

At last, we have the case when $(n-k) / p$ is not an integer. By the arguments in the first and second cases, after $O(p)+O([\log (n-k) / p \mid)$ time has passed, (4) becomes

$$
\begin{aligned}
& P_{n}=a_{1}+\frac{b_{2}}{a_{2}+} \frac{b_{3}}{a_{3}+} \cdots \frac{b_{k}}{a_{k}+X_{\lfloor(n-k) / p !}}, \\
& X_{\lfloor(n-k) / p]}=\left(\prod_{\substack{i=q \\
b(i)=1}}^{0}\left(c_{1}, c_{2}, c_{3}, c_{4}\right)^{2^{i}}, Y_{0}^{\prime}\right)=\left(h_{1}, h_{2}, h_{3}, h_{4}, X_{0}^{\prime}\right), \\
& X_{0}^{\prime}=\frac{e_{1}}{f_{1}+} \frac{e_{2}}{f_{2}+} \cdots \frac{e_{j}}{f_{j}+X_{0}} .
\end{aligned}
$$

The following main theorem summarizes the above results.

Theorem 3.1. Any periodic CF can be computed in $\mathrm{O}(\log n)$ time with $\mathrm{O}(1)$ space.

\section{Application examples}

In this section, we give examples to show the power of the method described in Section 3 . As the first example, the special quadratic surd number $\sqrt{7}$ can first be converted into the periodic $\mathrm{CF}$ $[2,1,1,1,4,1,1,1,4, \ldots]=[2, \overline{1,1,1,4}][6]$. Then using our aigorithm, a good approximation to $\sqrt{7}$ can be obtained in $O(\log n)$ time.

The second example is to solve the second-order linear recurrence $x_{n}=a x_{n-1}+b x_{n-2}$ with given coefficients $a, b$ and initial values $x_{0}, x_{1}$. By using the CF expansion of the ratio $x_{n} / x_{n-1}$ and the substitution scheme, we get

$$
\begin{aligned}
& \frac{x_{n}}{x_{n-1}}=a+\frac{b}{a+} \frac{b}{a+} \cdots \frac{b}{a+} \frac{b x_{0}}{x_{1}}=a+Y, \\
& Y=\frac{b}{a+} \frac{b}{a+} \cdots \frac{b}{a+Z}, \quad Z=\frac{b x_{0}}{x_{1}+x_{0}}
\end{aligned}
$$

The rational form oi $Y$ can be computed in $O(\log n)$ time. Then, it takes $O(1)$ time to obtain the rational form of $x_{n} / x_{n-1}$ and hence its numerator $x_{n}$. 
Corollary 4.1. The second-order linear recurrence $x_{n}=a x_{n-1}+b x_{n-2}$ can be solved in $\mathrm{O}(\log n)$ time.

The Fibonacci number $F_{n}$ is defined by

$$
F_{0}=0, \quad F_{1}=1, \quad F_{n}=F_{n-1}+F_{n-2} \text { for } n \geqslant 2 .
$$

The recurrence formula of the Fibonacci number is only a special case of the second-order linear recurrence where $a=1, b=1, x_{0}=0$ and $x_{1}=1$. (One can easily verify that $F_{n+1} / F_{n}$ equals the $n$th convergent of the simple continued fraction [1] . We therefore have the following corollary.

Corollary 4.2. The Fibonacci number $F_{n}$ can be computed in $\mathrm{O}(\log n)$ time.

\section{Concluding remarks}

Iñ this paper, a new substitution scheme is proposed to compute the CFs efficiently. We then embed a self-substitution concept into the scheme to design a general $O(\log n)$ algorithm for computing any periodic CF. In the past, many researchers $[3,4,5,8]$ have presented $O(\log n)$ algorithin: for computing $F_{n}$ based on the matrices-vector approach:

$$
\left(\begin{array}{c}
F_{n+1} \\
F_{n}
\end{array}\right)=\left(\begin{array}{cc}
1 & 1 \\
1 & 0
\end{array}\right)^{n}\left(\begin{array}{c}
F_{1} \\
F_{0}
\end{array}\right) .
$$

However, $F_{n}$ is just one instance of periodic CFs and most CFs cannot be computed using the matrices-vector approach.

Based on our substitution scheme, we have constructed a new parallel computation model for computing general CFs [1]. This model allows all prefix values of a CF to be computed in $O(\log n)$ time on a cost-optimal network in which $O(n / \log n)$ processors communicate via an inverse perfect shuffle routing mechanism.

\section{References}

[1] K.L. Chung, F.C. Lin and C.W. Chen, Parallel somputation of continued fractions, to be submitte1.

[2] L. Euler, De fractionibus continuis dissertatio, Personal manuscript, 1737 (An essay on continued fractions, translated by M.F. Wyman and B.F. Wyman, Math. Systems Theory 18 (1985) 295-328).

[3] D. Gries and G. Levin, Computing Fikonacci numbers (and sirularly defined functions) in $\log$ time, Inform. Process. Lett. 11 (2) (1980) 68-69.

[4] A.J. Martin and M. Rem, A representation of the Fibonacci algorithm, Inform. Process. Lett. 19 (2) (1984) 67-68.
[5] F.J. Lthinek, An $O(\log n)$ algorithm for computing the $n$th element of the solution of a difference equation, Inform. Process. Lett. 11 (2) (1980) 66-67.

[6] H.S. Wall, Analytic Theory of Continued Fractions (Van Nostrand, New York, 1948).

[7] J.B. Willian and W.J. Thron, Continued Fractions: Analytic Theory and Applicutions (Addison-Wesley, Reading, MA, 1980).

[8] T.C. Wilson and I. Shortt, An $O(\log n)$ algorithm for compuing general order-k Fibonacci numbers, Inform. Process. Lett. 10 (2) (1980) 68-75. 